\title{
Sprayable User Interfaces: Prototyping Large-Scale Interactive Surfaces with Sensors and Displays
}



Figure 1. Sprayable User Interfaces enable makers to create large-scale interactive surfaces on various materials and curved geometries. After designing an interactive artwork (a), our tool supports their fabrication with auto-generated stencils (b) enabling novel user interfaces that cover entire rooms (c), integrate in interactive architecture (d), and smart cities (e).

\section{ABSTRACT}

We present Sprayable User Interfaces: room-sized interactive surfaces that contain sensor and display elements created by airbrushing functional inks. Since airbrushing is inherently mobile, designers can create large-scale user interfaces on complex 3D geometries where existing stationary fabrication methods fail.

To enable Sprayable User Interfaces, we developed a novel design and fabrication pipeline that takes a desired user interface layout as input and automatically generates stencils for airbrushing the layout onto a physical surface. After fabricating stencils from cardboard or projecting stencils digitally, designers spray each layer with an airbrush, attach a microcontroller to the user interface, and the interface is ready to be used.

Our technical evaluation shows that Sprayable User Interfaces work on various geometries and surface materials, such as porous stone and rough wood. We demonstrate our system with several application examples including interactive smart home applications on a wall and a soft leather sofa, an interactive smart city application, and interactive architecture in public office spaces.

\section{Author Keywords}

Spraying; fabrication; printed electronics; ubiquitous computing; airbrush

Permission to make digital or hard copies of all or part of this work for personal or classroom use is granted without fee provided that copies are not made or distributed for profit or commercial advantage and that copies bear this notice and the full citation on the first page. Copyrights for components of this work owned by others than ACM must be honored. Abstracting with credit is permitted. To copy otherwise, or republish, to post on servers or to redistribute to lists, requires prior specific permission and/or a fee. Request permissions from Permissions@acm.org.

CHI '20, April 25-30, 2020, Honolulu, HI, USA

(C) 2020 Association for Computing Machinery.

ACM ISBN 978-1-4503-6708-0/20/04 ...\$15.00

https://doi.org/10.1145/3313831.3376249

\section{CSS Concepts}

- Human-centered computing Human computer interaction (HCI); Human-centered computing

\section{INTRODUCTION}

Since the early 1990s, Human-Computer Interaction researchers have envisioned a world in which digital user interfaces are seamlessly integrated with the physical environment until the two are indistinguishable from one another (Computer of the $21^{\text {st }}$ century [29]).

One of the greatest challenges in enabling this future is the integration of sensors and display elements with the physical environment, since the fabrication of interactive surfaces requires many design considerations, including how to adhere the elements to different materials and how to apply them onto irregular surface geometries in a manner accessible to novice users.

Over the last few years, novel fabrication methods have been developed that enable the fabrication of displays and sensors using inkjet- and screen-printing (PrintScreen [19]) as well as hydrographics (ObjectSkin [6]). However, all of these methods are limited to small-scale geometries, i.e. they are bound by the volume of the fabricating device, such as the size of the printer, the area of the screen-printing net, or the size of the hydrographic bath.

In this paper, we explore how to make large-scale user interfaces using spraying as the fabrication method. Unlike many existing techniques, such as 3D printing, screen printing or inkjet printing, spraying is not bound to a specific volume and, as often demonstrated by graffiti artwork, can create output that covers entire walls and even building facades. In addition, since spraying is a non-contact method, it works well on various surface textures (wallpaper, concrete, wood, bathroom tiles) and surface geometries, such as those with 
strong curvature (e.g., doubly curved geometries in modern architecture) and sharp angles (e.g., room/building corners).

To facilitate the design of Sprayable User Interfaces, we present a toolkit, which is integrated into a 3D editor and supports designers in creating artwork with interactive elements, such as integrated electroluminescent displays, proximity sensors and touch buttons and sliders. On export, the toolkit generates either a set of fabricated stencils (high-precision spraying on simple flat and singly curved surfaces) that the designer cuts from cardboard and adheres to the surface before spraying, or a set of projected stencils (less precise, but work for complex doubly-curved surfaces) that are displayed onto the surface via a projection pattern. After generating the stencils, designers spray each layer using a standard airbrush machine, attach a microcontroller to the user interface, and the interface is ready to be used.

Our work contributes to the vision of blending digital user interfaces with the physical environment and extends it to large-scale interactive surfaces. Since our method only uses DIY equipment, i.e. an airbrush system and a set of stencils, our work is readily accessible to HCI researchers and the maker community.

In summary, our core contributions are:

- $\square$ a new fabrication process for making large-scale interactive surfaces with display and sensor elements

- $\square$ a toolkit that supports makers in prototyping large-scale interactive surfaces, including the generation of stencils and instructions for spraying multiple functional layers

- $\square$ an evaluation of the conductivity of sprayed copper traces on different surface materials and geometries, and a user study on the precision of sprayed conductive traces when projected stencils rather than fabricated stencils are used

\section{RELATED WORK}

Our work is related to (1) fabricating methods for making small-scale sensors and displays (handheld, desktop-size), (2) painting and spraying as methods for making large-scale sensors and displays (room-sized), and (3) guidance systems that provide users with support while spraying.

Small-Scale Fabrication of Sensors and Displays (Inkjet Printing, Screen Printing, Hydrographics, 3D Printing)

Researchers have explored several methods to fabricate sensors and displays. Each of them supports various substrates and functional materials, however, all of them are limited in size to small-scale interactive elements since they are bound by the volume of the fabricating device.

Inkjet printing of functional inks (Instant Inkjet Circuits [12]) has been widely used to create sensors (Cuttable Multitouch Sensor [17]) and displays (PrintScreen [19]). However, inkjet printing is limited to materials that can pass through the print nozzle and substrates that can pass through the printer opening. In addition, the sensor/display size is limited to the area of the print bed.
Screen-printing offers greater flexibility in that is it compatible with a wider range of substrates that are not suitable for inkjet printing, such as stretchable silicone (Stretchis [30]), and works with a larger variety of materials, such as thermochromic or electroluminescent inks, which would otherwise block the inkjet printer nozzle. However, similar to inkjetprinting, the size of the sensor/display elements are limited to the area of the screen-printing net.

Another method for creating sensors and displays is hydrographics, which applies materials to a substrate by dipping it into a bath with a thin film of electronics that subsequently adheres to the surface (ObjectSkin [6]). While inkjetand screen-printing are 2D processes and either require folding (FoldIO [18]), transfer paper (SkinMarks [28]), or wrapping (Stretchis [30]) to create an interactive 3D surface, hydrographics can apply sensors and displays directly to a 3D geometry. However, it is also limited to small elements since the transferred electronics are bound by the size of the bath.

Finally, 3D printing, which has been used to create touch input (PrintPut [3]) and passive displays (Printed Optics [31], ColorMod [20]), is subject to the same limitation as the previously discussed methods, i.e. the size of the objects is limited by the available print volume.

\section{Large-Scale Fabrication of Sensors and Displays (Paint- ing, Spraying)}

While the fabrication output of the aforementioned methods is limited to small-scale elements, fabrication techniques, such as painting and spraying, in which the user freely moves around the fabrication 'head' (paint brush or spray nozzle) can cover large-scale surfaces.

To date, painting and spraying have mainly been used to create passive decorative artwork. Only recently, researchers have started to investigate how functional inks can be used to paint or spray sensors and displays: Wall ++ [33], FlexTouch [27], and Electrick [32] are examples for how conductive paint can be painted or sprayed onto walls to enable touch input via capacitive sensing. Living Wall [2] extends this work by embedding the conductive paint into a wallpaper that users can apply to room surfaces directly. To create large-scale passive displays via painting or spraying, researchers have explored the use of thermochromic (ShaderPrinter [22]) and photochromic (Photo-Chromeleon [9]) inks. Material scientists have also recently shown that it is feasible to spray electroluminescent inks [1],[4],[5],[23], which Lumilor [16] demonstrates by spraying active displays onto the surface of entire cars and motorbikes.

However, none of the above projects provide a toolkit that facilitates the creation of large-scale-user interfaces with user-configured shape and functionality, i.e. no tool is provided to guide makers in the process of creating interactive elements on a surface. In addition, none of the above approaches combine both visual output and touch sensing in one integrated workflow. 


\section{Training \& Guidance Systems for Spraying}

Achieving an even coverage using a spray-based method requires knowledge on how the spray paint behaves, i.e. at which distance, speed, and in which patterns to move the spray-can to achieve a desired result. To support novices in this process, researchers have developed virtual training systems with realistic simulations of the spray paint (viscosity, air pressure, paint pressure) to allow users to experiment with different spray patterns. While the first systems used VR and a mock-airbrush (Konieczny et al. [14]), later systems focused on increasing the authenticity by using AR and a realspraying device (Kim et al. [13]).

More recent systems have focused on supporting users in replicating an existing digital texture or painting: Luk et al.'s [15] and Shilkrot et al.'s [25] computer-controlled airbrush systems guide the user in spraying an image, i.e. the airbrush only sprays when the user holds their hand over the correct location. Similarly, SprayPrinter [10] is a handheld device which pairs a user's smartphone with a smart printer head that ejects paint in desired locations as it is moved across a surface. The images can be uploaded and printed without any artistic expertise. To bridge the gap between solely virtual and solely physical spraying, MobiSpray [24] uses a mobile phone as a virtual spray can and displays the resulting output via a projector that projects the 'spraying' result onto the physical object.

While all of the above systems centered on creating decorative artwork, we focus on interactive user interface elements, such as sensors and displays, which require more complex layering to achieve the desired functionality. Our work facilitates the creation of the 'functional artwork' by automatically generating stencils that guide the user in the process.

Finally, in addition to having users create the graffiti, researchers have also investigated how to automate the process using large-scale robotic systems. SprayPrinter [10] and PICARSO [8], for instance, are robots that can print murals of any size onto a flat surface. DroneGraffiti [26] is a flying graffiti system comprised of three aerial robots that operate in tandem to speed up the painting process. However, all of these systems require expensive hardware, i.e. robots or drones, whereas our approach can be carried out manually.

\section{SPRAYING USER INTERFACES}

In this paper, we explore the use of spraying as a fabrication technique for making large-scale interactive surfaces. These interactive surfaces contain input elements, such as touch sensors, sliders, and proximity sensors, as well as output elements, such as electroluminescent displays.

Spraying has several key benefits over existing fabrication methods, such as inkjet-printing and screen printing:

- $\square$ large-scale: as demonstrated by graffiti artists, spraying can cover large surfaces, such as entire walls or rooms, within a few hours
- $\square$ mobile: spraying hardware is portable; users can carry it around and create user interfaces on-the-go (Mobile Fabrication [21]) or on objects that cannot be moved

- $\square$ various geometries and materials: spraying enables the creation of user interfaces on a variety of different materials and on surfaces with complex or irregular geometry, such as those with double curvature

The remainder of the paper is structured as follows. We will first provide some background information on the hardware of our system, i.e. the airbrush and inks, and then provide details on the stencils and how they are used in the spraying process to create different interactive elements. Following this, we provide a walkthrough of our toolkit, detailing how users convert their digital design into a physical user interface and demonstrate this with an example of an interactive smart home application on a wall. We then show a series of additional applications, including an interactive room, an interactive public map sprayed on a real-life building, and a piece of interactive furniture. Finally, we conduct a technical evaluation of our spraying method, including a discussion of the challenges, and provide details on our implementation.

\section{HARDWARE: AIRBRUSH, INKS, AND SAFETY TOOLS}

Figure 2 shows our portable airbrush hardware. It consists of a mobile airbrush system, a set of functional inks required to create touch input and display elements, and basic safety tools for spraying.

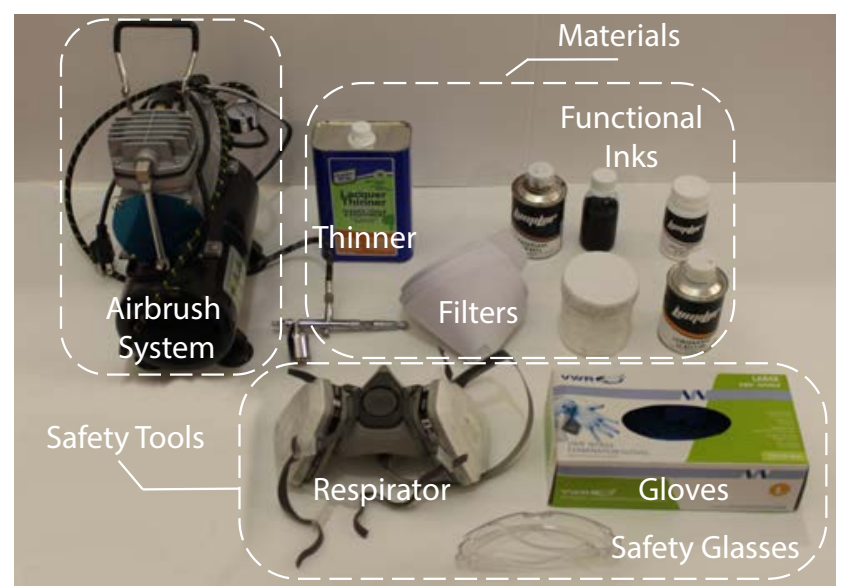

Figure 2. Our hardware setup includes an airbrush, functional inks, and safety tools.

We next provide background information on the choice of airbrush and types of functional inks.

Airbrush: Airbrushes are usually classified based on three characteristics: the trigger mechanism (double action, automatic, pistol grip or single action), the feed system (gravity fed, siphon fed or side feed) and the mix point, i.e. the point at which the air and paint mix; either inside the airbrush or externally after leaving the nozzle. For the trigger mechanism, we chose a double action because it simultaneously controls the paint volume and airflow which enables spraying fine patterns with less ink or large areas with more ink. For the feed system, we chose gravity-fed, which allows 
paint to be fed into the airbrush by its own weight. This is important for inks with large particles that tend to settle at the bottom of the container quickly. For the mix point, we chose an internal system which is more suited to fine details; systems with an external mix point are preferable for spraying large areas. Together, these configurations are most suitable for painting in fine detail, which is necessary for creating circuits. We use the airbrush with two different nozzles: a $0.35 \mathrm{~mm}$ nozzle for the clear conductor and dielectric ink, and $0.8 \mathrm{~mm}$ for the copper and phosphor inks. The wider nozzle for the copper and phosphor inks is preferable to prevent clogging due to the larger particle sizes.

Functional Inks: For spraying, inks must have a low viscosity appropriate to the nozzle size of the airbrush (i.e. thicker inks require a larger nozzle), or be thinned to achieve the required consistency. Any particles included must be small enough to ensure consistency in flow through the nozzle and to prevent clogging. In our system, we utilize copper, dielectric, phosphor, and clear conductor inks from Lumilor and KPT that are already prepared for usage in airbrush systems. Before using the inks, it is important to filter out bulks of particles that may form during storage. We therefore filter each ink through a 100 micron filter before use.

In detail, the functional inks we use are: copper ink from KPT (KPT EL-21) and Lumilor (Lumilor Backplane) that both have similar properties, dielectric ink from Lumilor, phosphor in blue/green from KPT (KPT EL41) and phosphor in orange from Lumilor (Lumilorange), as well as clear conductor (PEDOT:PSS) from KPT (KPT-EL5).

Safety Tools: Appropriate gloves, safety glasses and a respirator should be worn while spraying to avoid direct contact and inhalation of the inks.

\section{STENCILS: TYPES OF STENCILS \& SPRAYING ORDER}

While free-form spraying without stencils is popular with decorative artwork, spraying electronic circuits requires precision to avoid short circuits caused by overlapping between traces. Our toolkit supports fabricated stencils that offer high precision in spraying for electric components. Additionally, we offer a projection system to either display guidelines for placing the physical stencils or to display digital stencils on surfaces onto which physical stencils cannot be applied.

Single-/Multi-Layer Stencils (Type of Interactive Element) Figure 3 illustrates the stencil designs for our interactive elements. Most input elements, such as touch buttons, proximity sensors, and touch sliders require only a single layer of conductive ink. This is also the case for connector elements, such as wires and the microcontroller footprint that connects the sprayed user interface to the board that runs the code for sensing and visual output. A decorative artwork layer can be sprayed on top if desired. Electroluminescent touch-displays, in contrast, consist of 4 layers, i.e. a conductive bottom layer, dielectric layer, phosphor layer, and clear top conductor (PEDOT:PSS) (note that large displays require a conductive copper bus around the outlines of the display to distribute the energy evenly).



Figure 3. Input elements (touch buttons, sliders, proximity sensors) and connector elements (wires, microcontroller footprint) only require a single layer of conductive ink. Output elements, such as electroluminescent displays, require multiple layers.

Compound Stencils (Multi-Segment \& Multi-Element UI) For interactive elements that consist of multiple segments (e.g., a touch slider), we generate only a single stencil since all elements are sprayed from the same material (i.e., conductive ink). This is also the case if a user interface consists of multiple elements (e.g., if the interface has both a touch button and a touch slider, we generate only one stencil that contains both elements).

\section{Fabricated vs. Projected Stencils}

Fabricated stencils (Figure 4a) offer the highest precision but are limited to flat and single curved surfaces. For flat surfaces, stencils can be cut from rigid foam core or cardboard, whereas for single curved surfaces more flexible materials, such as vinyl sheets or adhesive foil can be used. To fabricate the stencils, users can use either a small cutting-plotter while on-the-go or a stationary laser cutter for larger elements.

For doubly-curved geometries, projected stencils (Figure 4b) can be used that visualize to the user where to spray the traces. While projected stencils offer less precision and require more expertise, the method is contactless and thus compatible with more surface geometries than physical stencils that need to be adhered to the surface. To project the stencils, users can use a regular sized or mini-projector.

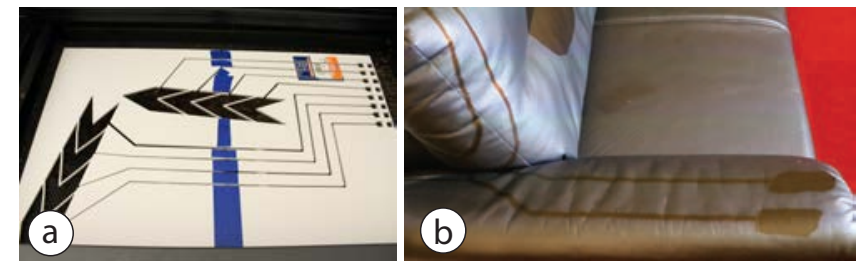

Figure 4. (a) Fabricated stencils. (b) Projected Stencil.

For both methods, if the stencil size exceeds the dimensions of the cutting device or projector, the stencils can be tiled into smaller partitions that can be cut on separate sheets or projected one after another. Once each stencil part is sprayed 
on the surface of the object, the resulting sprayed sensors/displays form one large element.

\section{Spraying Process and Stencil Order}

Users start by applying the first stencil onto the physical surface (i.e. either taping a fabricated stencil in place or by displaying a projected stencil). With the first ink loaded in the airbrush chamber, users then spray the first layer over the first stencil and remove the stencil. Each layer has to dry for 20 minutes before applying the next ink.

Before moving onto the next layer, which will be sprayed from a different material, users have to clean the airbrush and depending on the material used may have to switch the nozzle size. Once the next material is loaded into the airbrush, the next layer can be sprayed by repeating the above process.

Stencils are applied one after the other starting with the bottom most layer and then working the way up through the layer stack with the optional decorative artwork layer being sprayed last.

\section{TOOLKIT FOR SPRAYABLE USER INTERFACES}

Our toolkit that facilitates the design of Sprayable User Interfaces is integrated into the 3D editor Blender. It allows designers to add displays, touch, and proximity sensors, and touch sliders to 3D objects. Our design toolkit supports users during both the initial creation and subsequent design iterations of large-scale user interfaces.

In the following walkthrough, we illustrate how our toolkit supports artists by creating an interactive wall for controlling the room lights. It consists of one electroluminescent touch display (to turn the lights on and off), and two sliders (one for controlling hue, one for brightness). The final result is shown in Figure 13.

\#1 Creating a Virtual Spray Surface (3D Scan, 3D Model) Users start either by creating the digital model of their surface using the 3D editor's native modeling tools or by 3D scanning the surface in situ (e.g., using the 3D scanning capabilities of a mobile phone). For our walkthrough, since we are creating artwork for a flat wall, we measure the width and height of the wall and then create a matching 3D model in the $3 \mathrm{D}$ editor (Figure 5a).

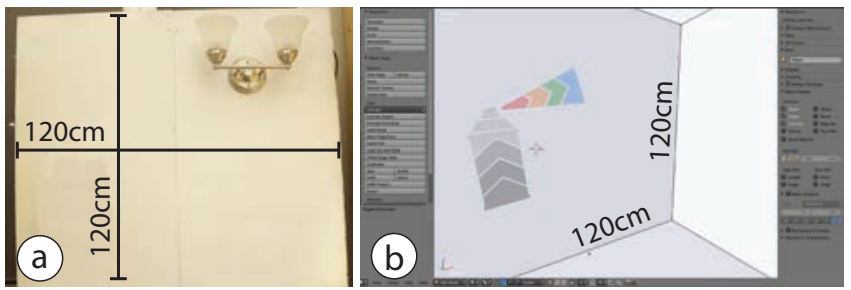

Figure 5. After measuring the physical wall (a), we recreate it in the 3D editor Blender. After designing our artwork in a 2D drawing tool, we import it into the 3D editor and apply it to the digital model.

Next, users create the visual artwork by sketching the design in a 2D drawing program (e.g., Adobe Photoshop) and then mapping the image as a texture onto the 3D model (e.g., using Blender's UV mapping tools). Here, we created a spray can design with a beam of color exiting the can. The body of the can will be used to control the brightness of an RGB lamp and the color beam will control its hue (Figure 5b).

\section{\#2 Adding User Interface Elements}

Users can select from 7 drawing tools that add interactivity. These include input elements, such as (1) touch buttons, (2) sliders (linear/free-form), and (3) proximity sensors; output elements, such as (4) electroluminescent displays; connector elements, such as (5) wires and (6) the microcontroller connector pattern; and (7) an erase tool to remove interactive elements.

Users can select each drawing tool by clicking on the corresponding button. They can then operate each tool in one of two different modes: free-form and vector-based. Electroluminescent displays can be drawn this way manually or imported from the design by using a color picker tool. The selected region from the artwork is then interpreted as a display.

We start by creating the two sliders for hue and brightness control: We click the 'linear slider' tool and draw a line corresponding to the slider length across the spray-can and another one across the exiting spray (Figure 6ab). We set the number of slider segments via the brush size. Next, we use the 'connector pattern' tool and place the connector at a conveninent location in the lower left corner of the wall and connect the slider segments to the connector using the 'wire' tool(Figure 6c).


Figure 6. Adding the hue and brightness sliders to the visual design: (a) Select linear slider tool; (b) Draw it onto the design; (c) Add wires and connector for the micro-controller.

While designing the UI, users can switch between visualizing only the artwork or only the interactive areas, or display both views overlapped (Figure 7).

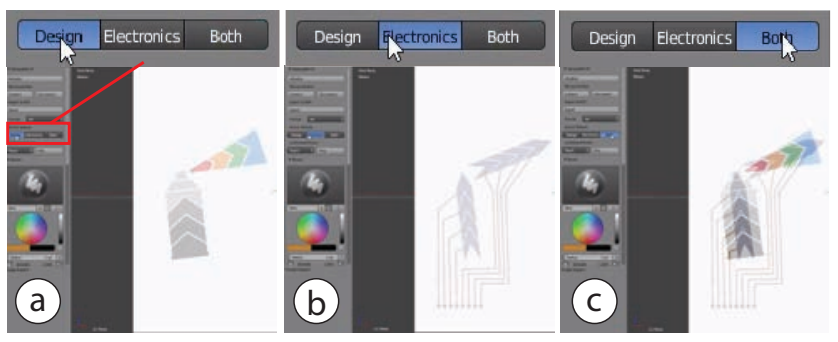

Figure 7. Switching between design and electronic views: The designer can view: (a) the design only, (b) the electronics only, or (c) overlap them. 


\section{\#3 Exporting the Stencils}

On export, the designer selects between fabricated or projected stencils. Fabricated stencils are exported as a set of 2D vector files (.pdf) that can be cut on a $2 \mathrm{D}$ cutting plotter or laser cutter (Figure 8). If the user interface requires multiple stencil layers, they are exported as separate vector files that can be cut individually. The projected stencils are exported as $2 \mathrm{D}$ images that can be projected onto the surface.

Since we spray onto a flat wall in our walkthrough example, we select fabricated stencils and cut them with a laser cutter.
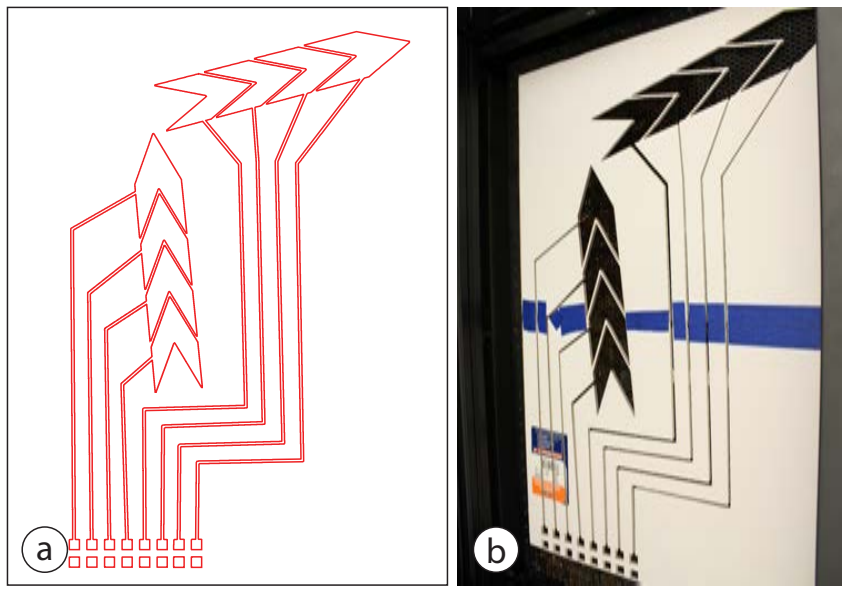

Figure 8. (a) The generated stencils are exported in .pdf format and (b) can be cut out with a laser cutter or a vinyl plotter. We glued two foam core sheets together

\section{\#4 Aligning and Applying the Stencils}

The first step when using stencils is to align them correctly on the surface. To do this, our toolkit generates stencil alignment guides that users can display with a projector. Before the guides can be displayed, users have to first calibrate the projector with the surface. To calibrate the projector, users measure the position of the projector relative to the surface, whereas the origin of the surface corresponds to the origin of its digital 3D model. After measuring the distance and angle of the projector with respect to the surface using a measurement tape and a protractor, users enter the data into the toolkit. Once the information is entered, users can click 'Project' and the first guide is mapped onto the physical surface, allowing users to align their stencil.

To display the guides for spraying the sliders, we place the projector at a distance of $3 \mathrm{~m}$ to the wall and at an angle of $0^{\circ}$, and, after aligning the projection, adhere the first stencil onto the wall.

\section{\#5 Spraying the User Interface}

Next, designers spray each layer of the design onto the surface. For our example, we start by spraying conductive copper ink for the sliders, the wires, and the micro-controller connector, remove the stencil and let it dry for 20 minutes. We next clean the airbrush and then spray the decorative artwork with its different hue and gray tones (Figure 9).

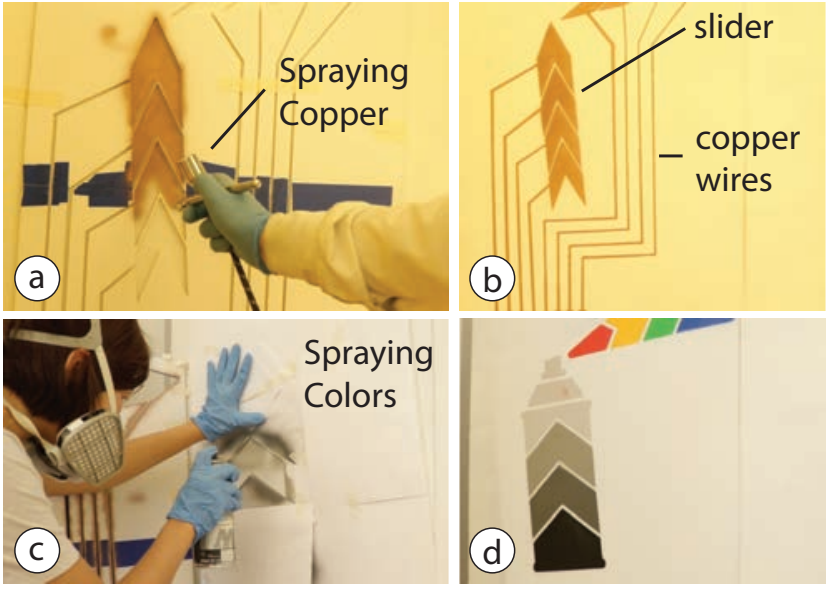

Figure 9. We first apply the stencil for the sliders and spray them with copper ink (a,b). Then, we spray our artwork with different colors over the sensors $(c, d)$.

\section{\#5 Connecting the Controller Board}

In the final step, designers attach the microcontroller to the wall using copper tape. To facilitate the development of Sprayable User Interfaces, we created the Graffiti Shield (Figure 10) that can be added to an Arduino Uno and contains all necessary electronics to control 6 touch buttons, sliders, or proximity sensors, and controls 2 electroluminescent touch displays. The code that runs on the Graffiti Shield controls the touch signals and the display segments over Serial communication, which can be used in application prototyping platforms, such as Processing.

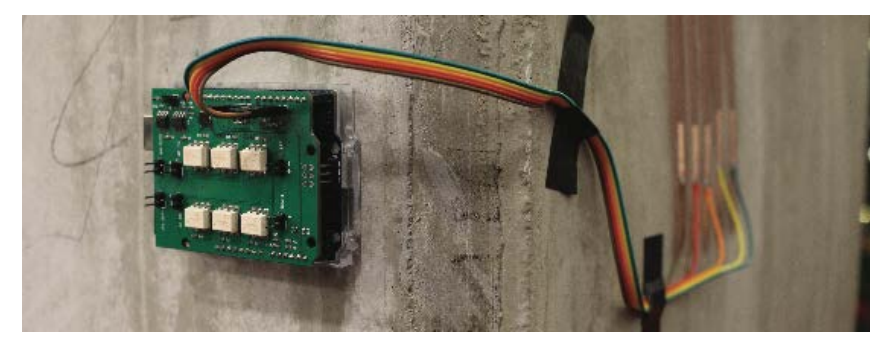

Figure 10. An Arduino Uno with our Graffiti Shield attached to the wall and connected to the sprayed connector pins from our interactive architecture example.

In our example, after we update the Graffiti Shield code to map input from the sliders with the hue/brightness control of the light and attach the Graffiti Shield to the sprayed microcontroller connector pins, our interface is ready to be used.

\section{\#6 Design Iteration: Add, Modify, Erase Elements}

Spraying enables us to add, modify or erase a user interface element. We can add a user interface element to an existing design by spraying additional layers. To modify or remove elements, we can either spray over them with the same color as the background or wipe them off the surface using a thinner. This allows users to quickly iterate over a design.

Adding an interactive element: Designers can add new components to an existing design by adding a new layer in the 3D editor. Designers can then choose to create the entire 
design as a stencil or only the new components. Continuing our walkthrough, after spraying the initial design, we notice we forgot a touch-button for turning the lights on/off. We first add it to the digital design (Figure 10a), fabricate the stencil and use the projected stencil to place the physical stencil at the correct position (Figure 10b), and finally spray the new sensor with conductive copper ink (Figure 10c).
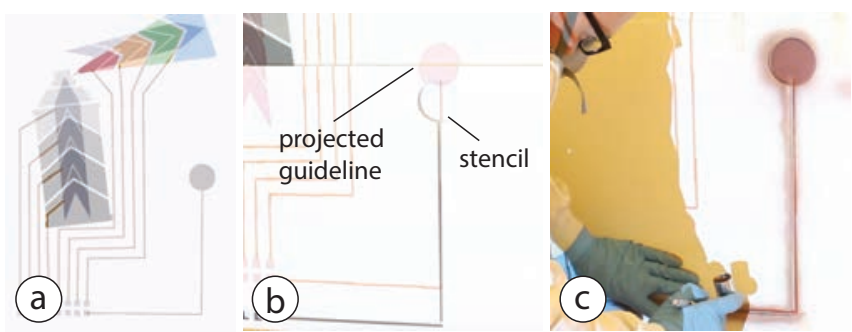

Figure 11. Adding components to the intermediate prototype: (a) A touch button added to the digital design; (b) Fabricated stencil is aligned using projected guide lines; (c) Spraying the additional sensor.

Erasing an interactive element: Connectors and interactive elements can be removed by first breaking the electronic connection using a lacquer thinner (Lean-Strip Green QKGL 75009) and then over-spraying the area in a color that matches the underlying surface. We tested several spray lacquers and found them to be well insulating (with the exception of carbon black paint which is conductive).

Continuing our walkthrough, after testing the touch button, we notice that it is too low and we need to adjust its position on the wall. After removing the touch button in the 3D editor (Figure 12a), we project the updated stencil onto the wall to show the areas that need to be removed (Figure 12b). Guided by the projection, we break the copper trace with lacquer (Figure 12c). Next, we spray over the rest of the touch button with white paint that matches the wall color (Figure 12d).We add a new touch button to the design as shown in the previous

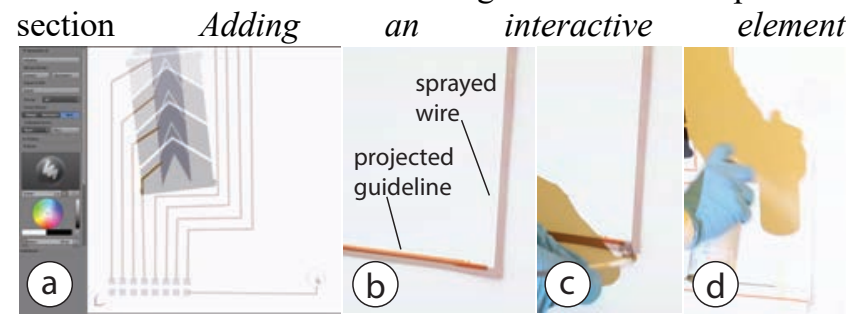

Figure 12. Erasing a component: (a) Remove touch sensor and wire from the digital model; (b) Project which areas need to be deleted; (c) Disconnect wire with lacquer thinner; (d) Overspray the remaining parts with white paint.

Modifying a Design: After using our interface, we notice that the touch button is difficult to find at night when the lights are off. We therefore decide to add an electro-luminescent display on top, effectively creating a touch-display. We first add the touch display in the digital editor and then transfer the design to the wall using the newly exported stencils. Figure 13 shows the final prototype with the electro-luminescent touch button used to turn the lights on/off.

\section{APPLICATION EXAMPLES}

Next, we present a range of application examples to illustrate the capabilities of our system.

\section{Smart Homes}

Spraying is particularly suitable to create large-scale content on walls or within entire rooms, enabling novel applications for smart homes, such as the light-control artwork described in the walkthrough: a blinking touch-display lets the user turn on the RGB lamp, sliding over the color beam changes the colors of the lights, sliding up and down on the spray can's body lets the user control the brightness. The design was created by spraying six stencil layers: (1) copper ink for the two sliders, wires, microcontroller connector, and touch button, (2) decorative artwork for the spray can and color beam consisting of a white primer and the visual design, (3 - 6) remaining layers for the touch display (dielectric, phosphor, copper bus, PEDOT:PSS). The sliders and the touch display are controlled with the Graffiti Shield. The total area of the design is $1.2 \times 1.2 \mathrm{~m}$.

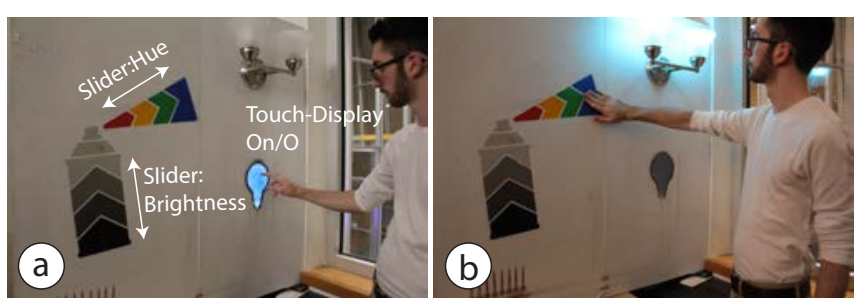

Figure 13. Smart Home Application: A touch display switches the ambient light in a room (a); The spray can artwork allows users to select the ambient color and brightness (b).

\section{Interactive Furniture}

Spraying is particularly suitable for large, doubly-curved objects that are difficult to augment with alternative printing methods (e.g., screen printing).

To illustrate this, we augmented a leather sofa with sensors and implemented a digital photo album that opens when a user sits and allows the user to swipe through the photos using mid-air gestures (Figure 14). When the user leaves the sofa, the app closes.

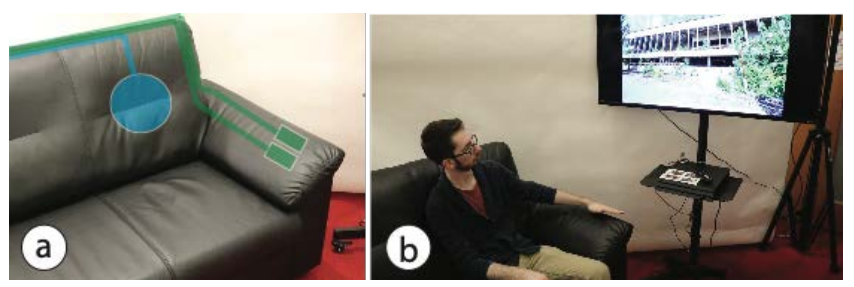

Figure 14. Interactive sofa. (a) A touch sensor (blue) detects if a users sits on the sofa. Two proximity sensors (green) sense swipe gestures. (b) A photo album app starts when a user sits down. Swiping over the armrest switches through photos.

To sense user interaction we integrated touch and proximity sensors into the sofa. The touch sensor on the backrest tracks if a user sits. The proximity sensors integrated with each arm rest sense mid-air gestures (swiping left and right). The sensors were sprayed with a single projected stencil and with the 
material PEDOT:PSS: a stretchable and clear conductive ink that seamlessly integrates into the sofa's material. The Graffiti board reads the signals from the touch sensor and the two capacitive proximity sensors and converts them into user present/not present and swipe right/left gesture commands. The total area of the element is $3.2 \mathrm{~m}^{2}$.

\section{Interactive Architecture in Public Spaces}

Spraying also allows users to augment large scale architecture with interactive elements. To illustrate this, we sprayed an interactive music control interface around a concrete pillar (Figure 15). The design consists of music notes in the form of a set of touch buttons that change the ambient music present in this space. The design was sprayed with two stencils: The projected stencil was used for the sensors and wires (1 layer), and fabricated stencils for the notes ( 1 layer, 4 colors). The Graffiti Shield reads the signal from each touch-button and converts it into a command to play a specific song. Note that due to the geometry and the material of the pillar, the sprayed elements had to be applied around sharp edges and on a porous surface, for which spraying is particular suited for. The total area of the element is $2.4 \mathrm{~m}^{2}$.

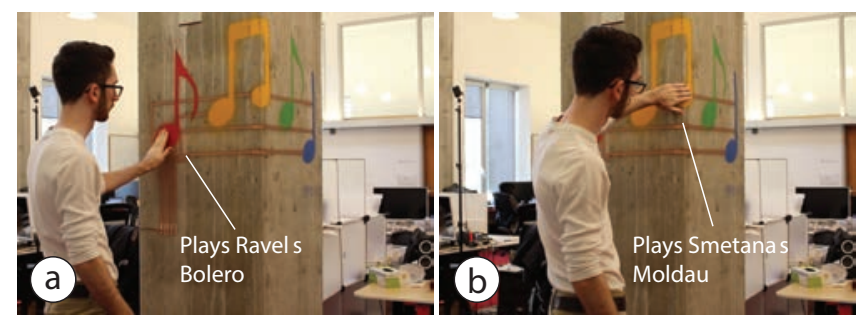

Figure 15. Ambient Music Interface on a concrete pillar with a porous surface: (a) Start song by touching a note; (b) Touch sensors even works on a pillar's sharp edge.

\section{Smart Cities}

Spraying as an inherently mobile fabrication technique allows interactivity to be added to immobile and large infrastructure elements in cities. We implemented a tourist guide (Figure 16) sprayed on a bollard that provides audible information on where to find the next subway station and guides to local attractions when users touch the icon-shaped EL displays.

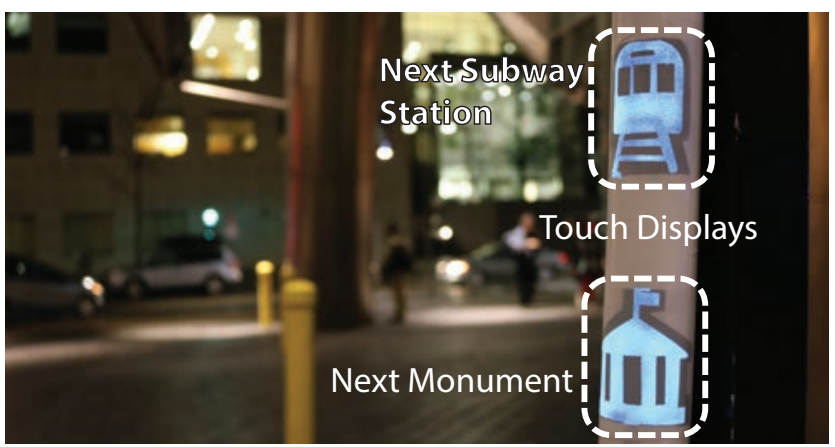

Figure 16. Smart cities. Curved touch displays give tourists audible information about sightseeing spots.
The graffiti board with an additional MP3 shield is connected to a speaker inside the bollard. All sprayed electronics are protected with an insulating clear coating and thus fully water resistant.

\section{TECHNICAL EVALUATION}

To evaluate spraying as a fabrication technique, we tested how well sprayable user interfaces work on (1) different materials, and (2) object geometries. We also performed a user study on the precision of sprayed traces when projected stencils are used.

\section{Experiment 1: Materials}

We hypothesized that creating user interfaces using a spraying technique works on a wider variety of surface materials than existing methods based on transfer paper (DuoSkin [11]) or hydrographics (ObjectSkin [6] ). Since transfer methods are contact-based, the copper particles on the transfer paper or film need to make sufficient contact with the object's surface, which is difficult for uneven geometries. In contrast, spraying is contactless, therefore, after exiting the airbrush, the copper particles can distribute freely on the object's surface and cover uneven surface textures with deeper cavities.

To evaluate this in practice, we repeated the experiment from Groeger et al. (ObjectSkin [6]) using the same types of materials (Figure 17). In addition, we also evaluated materials often used in architecture with more irregular or uneven surface geometries, such as porous stone (FireBricks) and cardboard. Since we use copper as the conductive material (rather than silver as in ObjectSkin [6]), we cannot compare the results directly, however, we can report relative values, i.e. a ranking of the materials by conductivity.

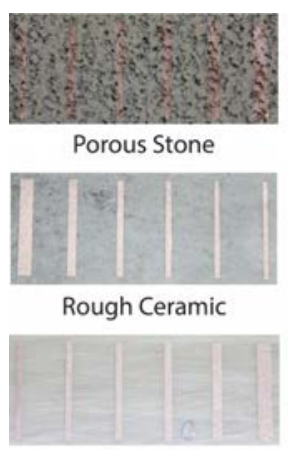

Transparent PVC

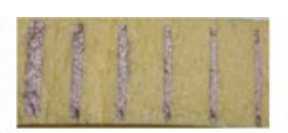

Organic Sponge

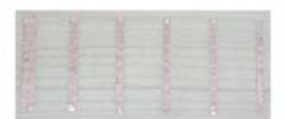

Rough Wood

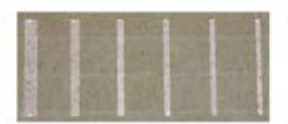

Cardboard

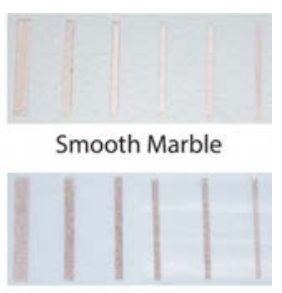

PLA

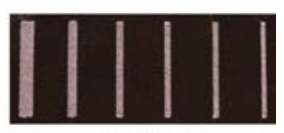

Leather
Figure 17. Conductive ink sprayed on substrates common in prototyping and architecture, $20 \mathrm{~mm}$ in length (trace widths, left to right: $2.6,1.6,1.2,1.0,0.9,0.8 \mathrm{~mm})$.

Similar to ObjectSkin [6], we only tested conductive paint in this evaluation as it is the most challenging to spray. Copper particles, unlike phosphor and dielectric inks, need to form a continuous surface to enable electric current to flow.

Apparatus: As outlined by Groeger et al. (ObjectSkin [6]), the primary indicator of the compatibility of the sprayed material and the substrate is the conductivity of the sprayed traces, which can be evaluated in terms of approximated sheet resistance [12]. We sprayed 6 samples of copper ink 
(KPT EL-21) with a length of $20 \mathrm{~mm}$ and varying width $(0.8$, $0.9,1.0,1.2,1.6,2.6 \mathrm{~mm}$ ) onto a sample of each material. We used adhesive vinyl sheets for the stencil (Figure 17). We then measured the resistance $R$ of each sample, and calculated the sheet resistance per square, $R_{s}$, according to the formula $R_{S}=R *$ (width/length). We averaged the results for each material.

Results: The results illustrated in Figure 18 show that all the copper traces sprayed exhibit high conductivity, regardless of the substrate material. The resistances range from $0.07 \Omega / \square$ to $0.25 \Omega /$, and include challenging materials like organic sponge and porous stone. Spraying achieved better results on all materials when compared to hydrographics (ObjectSkin), where the resistances ranged from $0.36 \Omega /$ to $1.45 \Omega /$ [ [11].

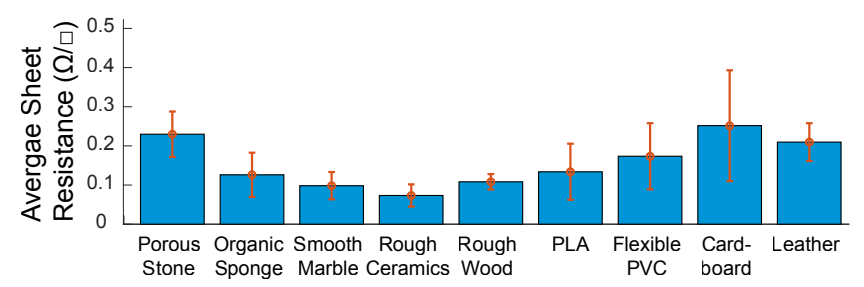

Figure 18. Average sheet resistance of copper traces on different substrate materials.

Figure 19 shows a micrograph of the porous stone, which was the surface with the highest degree of roughness. As can be seen in the magnified image, the copper particles are able to cover the entire surface, including deep and uneven cavities, resulting in high conductivity of the sprayed wires.

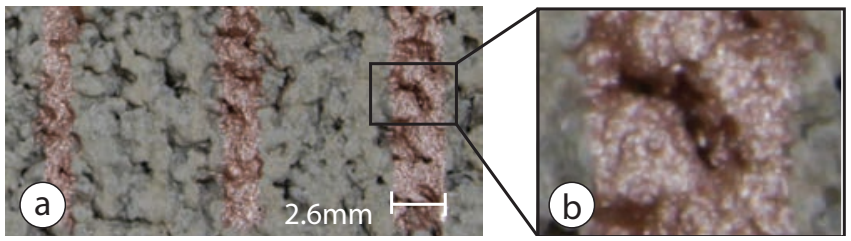

Figure 19. Sprayed conductive trace on porous stone (a).

Sprayed particles can reach deep cavities in the material and cover the entire material surface $(b)$.

In summary, both of the results from experiment \#1 show that spraying is suitable for a large variety of uneven and porous materials.

\section{Experiment 2: Complex Geometries}

Next, we evaluated how well spraying achieves an even coverage on sharp angles (e.g., room corners). We built our experiment onto Groeger et al. (ObjectSkin [6]). We extended their evaluation by covering outward and inward facing angles and used thinner traces to test the limits of spraying as a fabrication technique.

Apparatus: To test what angles can be supported during spraying, we printed four geometries with inward and outward facing angles, between $0^{\circ}$ and $135^{\circ}$, in $45^{\circ}$ increments. We then sprayed 6 lines, $20 \mathrm{~mm}$ in length and of varying widths: 0.8, 0.9, 1.0, 1.2, 1.6, $2.6 \mathrm{~mm}$, of conductive copper paint on each angle using an adhesive vinyl stencil, and spraying at a distance of approximately $15-20 \mathrm{~cm}$ from the surface, following the object's surface geometry (Figure 20). We measured the resistance per square of each sample using the same method as in the previous experiment, where the resistance was measured between the ends of each trace.

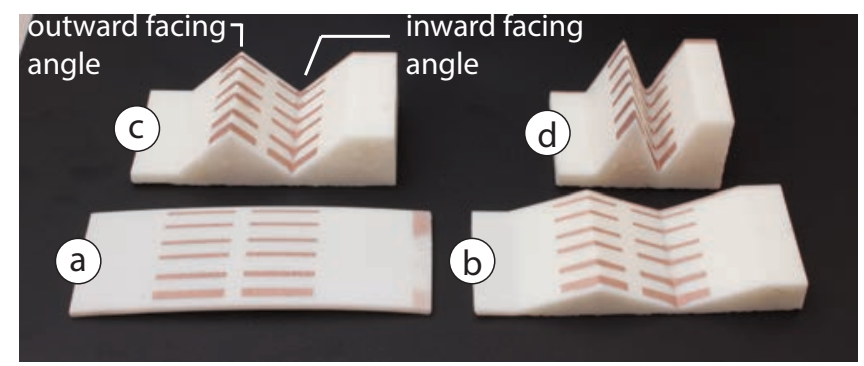

Figure 20. Conductive traces sprayed on geometries containing angles at (a) $0^{\circ}$, (b) $45^{\circ}$, (c) $90^{\circ}$ and (d) $135^{\circ}$.

Angle Results: We found that copper was conductive on all the angular geometries sprayed. Figure 21 shows measured results ranging from $0.15 \Omega /$ to $0.58 \Omega /$ and demonstrates that there is no significant relationship between the conductivity and the angle of the surface geometry. ObjectSkin [6] achieved a higher conductivity at $0^{\circ}$ for silver traces $(0.06 \Omega /)$; however, for inward facing angles above $30^{\circ}, \mathrm{Ob}-$ jectSkin's fabrication method resulted in a significant loss of conductivity, since the traces were printed on a hydrofilm which cracked when stretched too far. In contrast, we did not find any increase in resistance when spraying on steeper angles. The slight variance in resistance for our geometries can be explained by the manual nature of the fabrication process.

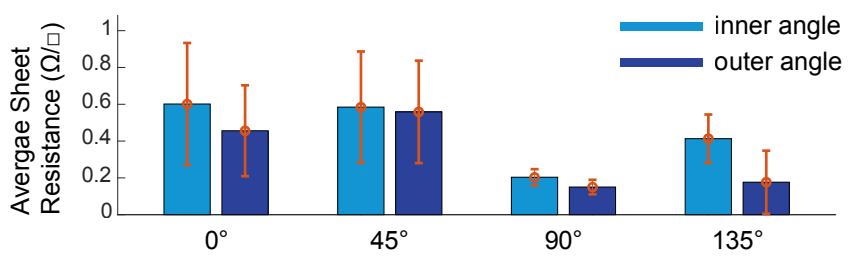

Figure 21. Average sheet resistance of copper traces for different surface geometries.

In summary, experiment \#2 shows that it is possible to augment unusual and novel geometric features with interactive surfaces, as illustrated by our application scenarios.

User Study: Precision of Traces with Projected Stencils Our system supports users in spraying on highly complex, doubly-curved surface geometries with a projection system. However, this requires the users to spray thin conductive traces without the aid of a fabricated stencil that provides a physical outline. We conducted a user study to investigate the extent to which users can spray thin traces using only a projected stencil.

Apparatus and Procedure: We asked 6 participants (2F) to spray 9 straight lines (40mm length) as thin as they can. 5 participants had no prior experience with airbrushing. We provided each participants with a sheet with 9 pre-printed black lines (40mm length) as guidelines for the spraying 
process (similar to how a projected stencil would show a guideline). We used the same airbrush as in the previous experiments (Iwata Eclipse $0.35 \mathrm{~mm}$ ). We began the study with a short demonstration on how to use an airbrush $(10 \mathrm{~min})$ and then allowed participants some time to practice spraying themselves (10min). After, users sprayed traces on the preprinted lines as thin as they were able to. Altogether, we collected 54 samples ( 9 samples x 6 participants).

Result: The average of the trace thickness was $2.11 \mathrm{~mm}$ (SD. $0.65 \mathrm{~mm}$ ) and the maximal distance between the printed line and a sprayed trace was $3.2 \mathrm{~mm}$. Thus, to ensure no intersection between two traces, their distance should be at least $6.4 \mathrm{~mm}$. Sprayed copper traces require a minimal thickness to be well conductive (as shown in the evaluation). Therefore, the limitation to about $2 \mathrm{~mm}$ thick traces does not impose a restriction of the design space.

\section{IMPLEMENTATION}

Our toolkit is a python plug-in for the 3D editor Blender.

\#1 Custom Brush Tools: We implemented a brush tool for each interactive element (touch buttons, sliders etc.). Each brush has its own unique color which enables the stencil generator

\#2 Converting 3D Geometry into 2D Fabricated Stencils: To generate flat $2 \mathrm{D}$ stencils from the $3 \mathrm{D}$ geometry, we first map the entire 3D geometry (surface + interactive elements) onto a 2D plane. For this, we use Blender's Paper Model add-on, which ensures that the aspect ratio of each polygon is preserved when unwrapping and that the model is separated into as few pieces as possible.

\#3 Extracting Interactive Areas for Stencils: Next, we extract the interactive elements from the 2D unwrapped texture. Since the interactive elements are colorful patches, we can use OpenCV's threshold and findContours() functions to find each UI element's outline in the texture. The contours of the elements are then drawn into a set of empty images, one for each stencil layer.

\#4 Exporting Stencil Cutting Files: Each image is then exported as a .pdf file using OpenCV's savePDF() and the .pdf files can then be cut from a 2D sheet using a cutting plotter. To help users spray the layers in the correct order, our plugin generates the layers with filenames corresponding to the respective layer (e.g. layer_1_copper.pdf).

\#5 Projection Mapping: When users hit 'Project', our 3D editor plug-in computes a projection mapping from the virtual camera position to the digital 3D model. The projection compensates for the object's geometry and can therefore render a surface texture on any geometry except when occlusion occurs. Our implementation generates the projection in under one minute for our current projector (AAXA M6, 1080p).

\#6 Sensing and Display Driver: Touch and proximity sensing are implemented using Arduino's capacitive sensing library. Mid-air swiping uses 2 proximity sensors and detects which sensor is triggered first to identify the gesture's direction. The electroluminescent displays are powered with $12 \mathrm{~V}$ that are converted to $220 \mathrm{~V}$ AC with an inverter (PYLELI-ISC) and sense touch using time-multiplexing [30] and capacitive sensing.

Note on Electro-Luminescent Display Layers: For electroluminescent displays, the extracted contour (step \#3) is further split into a set of layers, i.e. for each layer a new empty image is created and the contour is scaled according to the specification (copper layer: original shape, subsequent layers: expand original shape by $5 \mathrm{~mm}$ in each normal direction to cover the copper. Copper frame: $1 \mathrm{~cm}$ wide frame created by upscaling the original contour by $10 \mathrm{~mm}$ in normal direction).

\section{DISCUSSION}

In this section, we discuss limitations and future opportunities for sprayable user interfaces.

Displays on rough materials: Layers of an electroluminescent display must have an even thickness to prevent shortcuts and provide even brightness. Smooth surfaces are necessary to achieve such layers. Rough surfaces (like the porous stone from the evaluation section) require a foundation prior to the display spraying. Small irregularities can be evened out with a clear coating spray. Very rough materials have to be sanded down or require a foundation such as plaster.

Stencils: Since the stencils need to be computationally generated in advance, the user has to create their design first in a digital editor, which reduces the opportunity for spontaneous exploration on the physical surface. Creating the stencils also requires additional time, material, and hardware, and aligning the stencils can be tedious. Future work could explore the use of modular stencils for UI elements (e.g., a set of stencils that create touch buttons of different sizes) or shape-changing stencils that adjust themselves based on a desired user interface shape.

\section{CONCLUSION}

We presented Sprayable User Interfaces; room-sized interactive surfaces with sensor and display elements created by spraying functional inks. We showed the benefits of spraying over traditional fabrication methods for making interactive surfaces, i.e. spraying is mobile and allows user interfaces to be created even in environments where existing stationary fabrication methods fail. We demonstrated our design and fabrication pipeline that supports users in stencil generation and included a projection system for complex geometries. We provided results from our technical evaluation, showing that Sprayable User Interfaces can be created on a wide range of surface materials and geometries (e.g. concrete buildings, room corners), in sizes up to several square meters. For future work, we plan to expand the current fabrication capabilities, enabling further user elements to be added to our toolkit, such as thermo- and electrochromic displays.

\section{ACKNOWLEDGMENT}

We thank the Engineering and Physical Sciences Research Council (EPSRC EP/N509619/1) for partially funding this work. 


\section{REFERENCES}

[1] Mariya Petrova Aleksandrova. Improvement of the electrical characteristics of polymer electroluminescent structures by using spray-coating technology. In Journal of Coatings Technology and Research 9, 2, 157161, 2012.

[2] Leah Buechley, David Mellis, Hannah Perner-Wilson, Emily Lovell, and Bonifaz Kaufmann. 2010. Living wall: programmable wallpaper for interactive spaces. In Proceedings of the 18th ACM international conference on Multimedia (MM '10). ACM, New York, NY, USA, 1401-1402.

[3] Justin Burstyn, Nicholas Fellion, Paul Strohmeier, and Roel Vertegaal. PrintPut: Resistive and Capacitive Input Widgets for Interactive 3D Prints. In Proceedings of INTERACT 2015. Springer Berlin Heidelberg, 2015, 332-339.

[4] Aubrey L. Dyer, Emily J. Thompson, and John R. Reynolds. Completing the Color Palette with SprayProcessable Polymer Electrochromics. In American Chemical Society Applied Materials \& Interfaces 3, 6, 1787-1795, 2011.

[5] Katsuhiko Fujita, Takamasa Ishikawa, and Tetsuo Tsutsui. Separate-Coating and Layer-by-Layer Deposition of Polymer Emitting Materials by the Spray Deposition. In Molecular Crystals and Liquid Crystals 405, 1, 83-88, 2003.

[6] Daniel Groeger and Jürgen Steimle. ObjectSkin: Augmenting Everyday Objects with Hydroprinted Touch Sensors and Displays. In Proceedings of the ACM on Interactive, Mobile, Wearable and Ubiquitous Technologies 1, 4, Article 134, 2018.

[7] David Holman and Roel Vertegaal. Organic User Interfaces: Designing Computers in Any Way, Shape, or Form. In Communucations of the ACM 51, 6, 48-55.

[8] Ian Hooi, Samuel Oosterholt, Sven Paschburg, Joyce Phan, Neil Yeoh, Ben Cazzolato. PICARSO: Programmable Interface Controller with Autonomous Robotic Spraying Operation. 2010.

[9] Yuhua Jin, Isabel Qamar, Michael Wessely, Aradhana Adhikari, Katarina Bulovic, Parinya Punpongsanon, and Stefanie Mueller. 2019. Photo-Chromeleon: ReProgrammable Multi-Color Textures Using Photochromic Dyes. In Proceedings of the 32nd Annual ACM Symposium on User Interface Software and Technology (UIST '19). Association for Computing Machinery, New York, NY, USA, 701-712.

[10] Mihkel Joala. SprayPrinter. 2017. https://newatlas.com/sprayprinter-wall-sized-graffiti-art/51019/

[11] Hsin-Liu (Cindy) Kao, Christian Holz, Asta Roseway, Andres Calvo, and Chris Schmandt. DuoSkin: rapidly prototyping on-skin user interfaces using skin-friendly materials. In Proceedings of the 2016 ACM
International Symposium on Wearable Computers (ISWC '16), 16-23.

[12] Yoshihiro Kawahara, Steve Hodges, Benjamin S. Cook, Cheng Zhang, and Gregory D. Abowd. Instant Inkjet Circuits: Lab-based Inkjet Printing to Support Rapid Prototyping of UbiComp Devices. In Proceedings of the 2013 ACM International Joint Conference on Pervasive and Ubiquitous Computing

(UbiComp'13), 363-372.

[13] Daeseok Kim, Youngwoo Yoon, Sunyu Hwang, Geehyuk Lee, and Jinah Park. Visualizing Spray Paint Deposition in VR training. In Proceedings of the IEEE Virtual Reality Conference (VR'07). 307-308.

[14] Jonathan Konieczny, John Heckman, Gary Meyer, Mark Manyen, Marty Rabens, and Clement Shimizu. Automotive Spray Paint Simulation. In Advances in Visual Computing (ISVC'08), 998-1007.

[15] Joseph Luk. Computer-Controlled Airbrush with Motion Tracking. Technical Report, University of British Colombia, 2004.

[16] LumiLor: Light Emitting Coating. https://www.lumilor.com/

[17] Simon Olberding, Nan-Wei Gong, John Tiab, Joseph A. Paradiso, and Jürgen Steimle. A Cuttable MultiTouch Sensor. In Proceedings of the 26th Annual ACM Symposium on User Interface Software and Technology (UIST '13), 245-254.

[18] Simon Olberding, Sergio Soto Ortega, Klaus Hildebrandt, and Jürgen Steimle. Foldio: Digital Fabrication of Interactive and Shape-Changing Objects With Foldable Printed Electronics. In Proceedings of the 28th Annual ACM Symposium on User Interface Software \& Technology (UIST '15), 223-232.

[19] Simon Olberding, Michael Wessely, and Jürgen Steimle. PrintScreen: Fabricating Highly Customizable Thin-Film Touch-Displays. In Proceedings of the 27th Annual ACM Symposium on User Interface Software and Technology (UIST'14), 281-290.

[20] Parinya Punpongsanon, Xin Wen, David S Kim, and Stefanie Mueller. ColorMod: Recoloring 3D Printed Objects using Photochromic Inks. In Proceedings of the 2018 CHI Conference on Human Factors in Computing Systems (CHI'18), 213.

[21] Thijs Roumen, Bastian Kruck, Tobias Dürschmid, Tobias Nack, and Patrick Baudisch. Mobile Fabrication. In Proceedings of the 29th Annual Symposium on User Interface Software and Technology (UIST '16), 3-14.

[22] Daniel Saakes, Masahiko Inami, Takeo Igarashi, Naoya Koizumi, and Ramesh Raskar. Shader printer. In $A C M$ SIGGRAPH 2012 Emerging Technologies (SIGGRAPH '12), Article 18.

[23] Andreas Sandström, Amir Asadpoordarvish, Jenny Enevold, and Ludvig Edman. Spraying Light: 
Ambient-Air Fabrication of Large-Area Emissive Devices on Complex-Shaped Surfaces. In Advanced Materials 26, 29, 4975-4980, 2014.

[24] Jürgen Scheible and Timo Ojala. MobiSpray: Mobile Phone as Virtual Spray Can for Painting BIG Anytime Anywhere on Anything. Leonardo, 42(4), 332-341, 2009.

[25] Roy Shilkrot, Pattie Maes, Joseph A. Paradiso, and Amit Zoran. Augmented Airbrush for Computer Aided Painting (CAP). In ACM Transactions on Graphics 34, 2, Article 19, 2015.

[26] Anastasia Uryasheva, Mikhail Kulbeda, Nikita Rodichenko, and Dzmitry Tsetserukou. 2019. DroneGraffiti: autonomous multi-UAV spray painting. In ACM SIGGRAPH 2019 Studio (SIGGRAPH '19). ACM, New York, NY, USA, Article 4, 2 pages.

[27] Yuntao Wang, Jianyu Zhou, Hanchuan Li, Tengxiang Zhang, Minxuan Gao, Zhuolin Cheng, Chun Yu, Shwetak Patel, and Yuanchun Shi. 2019. FlexTouch: Enabling Large-Scale Interaction Sensing Beyond Touchscreens Using Flexible and Conductive Materials. Proceedings on the ACM Interactive Mobile, Wearable and Ubiquitous Technologies. 3, 3, Article 109 (September 2019), 20 pages.

[28] Martin Weigel, Aditya Shekhar Nittala, Alex Olwal, and Jürgen Steimle. SkinMarks: Enabling Interactions on Body Landmarks Using Conformal Skin Electronics. In Proceedings of the 2017 CHI Conference on
Human Factors in Computing Systems (CHI '17), 3095-3105.

[29] Mark Weiser. The Computer for the 21st Century. In Mobile Computing Communications (SIGMOBILE) Rev. 3, 3, 3-11, 1999.

[30] Michael Wessely, Theophanis Tsandilas, and Wendy E. Mackay. Stretchis: Fabricating Highly Stretchable User Interfaces. In Proceedings of the $29^{\text {th }}$ Annual Symposium on User Interface Software and Technology (UIST16), 697-704.

[31] Karl Willis, Eric Brockmeyer, Scott Hudson, and Ivan Poupyrev. Printed Optics: 3D Printing of Embedded Optical Elements for Interactive Devices. In Proceedings of the 25th Annual ACM Symposium on User Interface Software and Technology (UIST'12), 589-598.

[32] Yang Zhang, Gierad Laput, and Chris Harrison. 2017. Electrick: Low-Cost Touch Sensing Using Electric Field Tomography. In Proceedings of the 2017 CHI Conference on Human Factors in Computing Systems (CHI '17). ACM, New York, NY, USA, 1-14.

[33] Yang Zhang, Chouchang (Jack) Yang, Scott E. Hudson, Chris Harrison, and Alanson Sample. 2018. Wall++: Room-Scale Interactive and Context-Aware Sensing. In Proceedings of the 2018 CHI Conference on Human Factors in Computing Systems (CHI '18). ACM, New York, NY, USA, Paper 273. 